\title{
Knowledge on Hazards of Smok- ing and Benefits of Quitting Smoking among the Smokers
}

Keywords: Smoking; Hazards of smoking; Quitting smoking; Smokers Abstract

A study to assess the knowledge on hazards of smoking and benefits of quitting smoking, among smokers admitted in Rajah Muthiah medical college hospital, Chidambaram. The quantitative research approach was used to assess the knowledge on hazards of smoking and benefits of quitting smoking among the smokers. A descriptive cross sectional survey was adopted and conducted at Rajah Muthiah medical college hospital among 463 samples, Chidambaram. The target population refers to patients who have the habit of smoking. Smokers, aged 20 years and above, who fulfilled the inclusion criteria were the subjects selected for the study. Simple random sample technique was used in selecting samples from the smokers admitted in medical, surgical, and orthopaedic wards. The study subjects were interviewed with closed ended questionnaire, which was prepared by the investigator to assess the knowledge regarding hazards of smoking and benefits of Quitting smoking. The statistical tests, both descriptive and inferential, were used for analyzing the data. The study results revealed that the knowledge on the hazards of smoking was moderately adequate and benefits of quitting was adequate among most of the subjects. The level of nicotine dependency showed low nicotine dependence among most of the subjects, and the level of temptation showed extreme temptation to smoke due to various situations. Most of the subjects were in contemplation and preparation stage of readiness to quit.

\section{Introduction}

Smoking is marketed as a masculine habit, linked to health, happiness, fitness, power, and virility. People start to smoke because of curiosity, or as they feel it, as a glamorous, exciting, stress reducing activity. Later, because of peer pressure and the tobacco industry's advertisements and promotions for its products, tobacco usage turns into tobacco addiction over a period of years in an individual's life. In reality, it leads to sickness, premature death, and infertility. Tobacco use is a risk factor for 6-8 leading cause of death in the world. Tobacco, no doubt is the most common cause of the death in the world. Tobacco kills more than 5 million people which are much more than HIV/AIDS, tuberculosis, malaria combined. By 2030 tobacco is going to kill 8 million people a year [1].

Global Health Observatory, WHO, has estimated that about 14.5 million total deaths occurred in 2008 in South East Asian Region (SEAR) [2]. Out of this, 7.9 million (55\%) were due to noncommunicable diseases. Cardiovascular Disease (CVDs) alone accounted for $25 \%$ of all deaths. Chronic respiratory disease (CRDs), cancer, and diabetes accounted for $9.6 \%, 7.8 \%$ and $2.1 \%$ of all deaths respectively. In the developed countries, the prevalence of smoking for males and females is $39 \%$ and $24 \%$ respectively. Whereas, in the developing countries the gender difference is larger with $49 \%$ of males and only $7 \%$ of females [1]. The findings from Global Youth Tobacco
Journal of

\section{Addiction \& Prevention}

\author{
Sara B Reader*and Vijayalakshmi Ethiraj \\ Rani Meyyamai College of Nursing, India \\ *Address for Correspondence \\ Sara B Reader, Rani Meyyamai College of Nursing, India, E-mail: \\ sarasrinisha@yahoo.com \\ Submission: 28 August, 2018 \\ Accepted: 14 September, 2018 \\ Published: 20 September, 2018 \\ Copyright: () 2018 Reader SB, et al. This is an open access article \\ distributed under the Creative Commons Attribution License, which \\ permits unrestricted use, distribution, and reproduction in any medium, \\ provided the original work is properly cited.
}

Survey among youths (15-24 years) in Chandigarh revealed that the prevalence of current smoking among youths was $20.4 \%$ in males. Smoking peers, cigarette advertisements, and feeling comfortable in social gatherings were significant determinants for smoking [3].

Hence, the researcher decided to focus "A study to assess the knowledge on hazards of smoking and benefits of quitting smoking, among smokers admitted in Rajah Muthiah Medical College Hospital, Chidambaram".

Objective: To assess the knowledge on hazards of smoking and benefits of quitting smoking among the smokers.

\section{Materials and Methods}

The quantitative research approach was used to assess the knowledge on hazards of smoking and benefits of quitting smoking among the smokers. A descriptive cross sectional survey was adopted and conducted at Rajah Muthiah Medical College Hospital among 463 samples, Chidambaram. The target population refers to patients who have the habit of smoking. Smokers, aged 20 years and above, who fulfilled the inclusion criteria were the subjects selected for the study. Simple random sample technique was used in selecting samples from the smokers admitted in medical, surgical, and orthopaedic wards. Closed ended questionnaire was developed by the researcher to assess the knowledge on hazards of smoking. It consisted of 12 statements pertaining to smoking related disorders with 3 options as Yes/No/ Do not know. A closed ended questionnaire was prepared by the investigator to determine the benefits of quitting in terms of reduced risk of getting tobacco related disorders for one self and others. The response was tested as Yes/No/Do not know. The data collection was done from June 2009 to March 2010. The study subjects were interviewed knowledge regarding hazards of smoking and benefits of quitting smoking. The descriptive statistical tests, Percentage were used for analyzing the data.

\section{Results}

Table 1 shows the distribution of knowledge of the subjects on the hazards of smoking. The number of subjects who had awareness about hazards of smoking on health as lung problems by 356 (76.89\%), as heart attack/myocardial infarction by 309 (66.74\%) subjects, as cancer by 307 (66.31\%) of the subjects, and as oral/dental 
Table 1: Distribution of knowledge response on the hazards of smoking among the subjects $(\mathrm{N}=463)$.

\begin{tabular}{|c|c|c|c|c|c|c|c|}
\hline \multirow{2}{*}{ Items } & \multirow{2}{*}{ Hazards of smoking } & \multicolumn{2}{|c|}{ Yes } & \multicolumn{2}{|c|}{ No } & \multicolumn{2}{|c|}{ Don't Know } \\
\hline & & No. & $\%$ & No. & $\%$ & No. & $\%$ \\
\hline $\mathrm{K} 1$ & Lung problems & 356 & 76.89 & 21 & 4.54 & 86 & 18.57 \\
\hline $\mathrm{K} 2$ & Vascular problem in leg & 195 & 42.1 & 73 & 15.78 & 195 & 42.11 \\
\hline K3 & Heart attack/ myocardial infarction & 309 & 66.74 & 45 & 9.72 & 109 & 23.54 \\
\hline K4 & Cancer & 307 & 66.31 & 53 & 11.45 & 103 & 22.3 \\
\hline K5 & Oral/dental problems & 297 & 64.15 & 69 & 14.9 & 97 & 20.95 \\
\hline K6 & Reduces longevity of life & 231 & 49.89 & 92 & 19.87 & 140 & 30.24 \\
\hline K7 & Contain $4000+$ chemicals & 114 & 24.62 & 70 & 15.12 & 279 & 60.26 \\
\hline $\mathrm{K} 8$ & Carcinogens & 175 & 37.8 & 68 & 14.69 & 220 & 47.52 \\
\hline K9 & Infertility & 142 & 30.67 & 103 & 22.25 & 218 & 47.08 \\
\hline K10 & Hypertension & 207 & 44.78 & 77 & 16.63 & 179 & 38.66 \\
\hline K11 & Gastric problems & 195 & 42.12 & 80 & 17.3 & 188 & 40.61 \\
\hline $\mathrm{K} 12$ & Poor Health impact on others & 256 & 55.29 & 73 & 15.77 & 134 & 28.94 \\
\hline
\end{tabular}

problems by 297 (64.15) of them. Among the subjects, 268 (57.89\%) had lack of awareness regarding peripheral vascular problems in leg, $349(75.38 \%)$ subjects did not know that cigarette contains $4000+$ chemicals, $288(62.21 \%)$ subjects did not know that cigarette has carcinogens, and $321(69.33 \%)$ subjects were unaware about smoking as a risk factor for occurrence of infertility. Nearly half of the subjects had awareness that smoking acts as risk factor for hypertension, reduces longevity of life, and leads to poor health impact on others around them.

Table 2: Distribution of level of knowledge regarding hazards of smoking among the subjects $(\mathrm{N}=463)$.

\begin{tabular}{|c|c|c|c|}
\hline \multicolumn{2}{|c|}{ Knowledge on hazards smoking } & \multirow{2}{*}{ Number } & Percentage \\
\hline Score Range & Level of knowledge & & 36.7 \\
\hline 0-4 & Inadequate & 170 & 37.4 \\
\hline 8-May & Moderate & 173 & 25.9 \\
\hline 12-Sep & Adequate & 120 & \\
\hline
\end{tabular}

The above table predicts the distribution of level of knowledge regarding ill effects of smoking among 463 subjects. Among the subjects, 170 (36.7\%) had inadequate knowledge, 173 (37.4\%) of the subjects had moderately adequate knowledge, and 120 (25.9\%) subjects had adequate knowledge on hazards of smoking. The minimum score of ' 0 ' was obtained by 22(4.75\%) of the study subjects and maximum score of ' 12 ' was obtained by 46 (9.94\%) study subjects.

Table 3 explained about the distribution of knowledge response regarding benefits of quitting. Out of $463,363(78.40 \%)$ of the subjects had awareness about the benefits of quitting smoking as improvement in general health and reduction in heart problems by $317(68.48 \%)$ subjects, as paving way for saving money by 305 (65.87\%) subjects, as increasing happiness in the family by 306 (66.69\%) of subjects, and as reduction of respiratory problems by $304(65.66 \%)$ of them. There was lack of knowledge on reduction of vascular problems in the leg by 298 (64.37\%) subjects, and 205 (44\%) subjects were unaware that improvement in peace of mind happens as a result of quitting.

Table 4 depicts the distribution of level of knowledge of the subjects regarding benefits of quitting smoking. About 219 (47.3\%) subjects had adequate knowledge on the benefits of quitting smoking, $158(34.1 \%)$ subjects had inadequate knowledge on benefits of quitting, and $86(18.6 \%)$ subjects had moderate knowledge regarding benefits of quitting smoking. The minimum score of ' 0 ' was obtained by $21(4.54 \%)$ subjects and maximum score of 10 was obtained by 144 (31.10\%) study subjects.

\section{Discussion}

This study found that based on 12 items regarding knowledge on hazards of smoking among 463 study participants majority of the subjects had awareness about hazards of smoking such as occurrence of lung problems (76.89\%), myocardial infarction (66.74\%), cancer (66.31\%), and dental problems (64.15\%). Majority of the subjects had lack of knowledge related to hazards of smoking such as $4000+$ chemicals in cigarettes $(75.38 \%)$, carcinogens $(62.21 \%)$, risk factor for infertility (69.33\%), and occurrence of peripheral vascular problems among $268(57.89 \%)$ of the subjects. The overall knowledge score reflects that $36.7 \%$ of the subjects had inadequate knowledge and $37.4 \%$ had moderately adequate knowledge and only $25.9 \%$ of them had adequate knowledge. Thus the findings suggest that there was lack of awareness on hazards of smoking among the smokers. These findings are supported by the following studies.

Table 3: Distribution of knowledge response regarding benefits of quitting among the subjects $(\mathrm{N}=463)$.

\begin{tabular}{|c|c|c|c|c|c|c|c|}
\hline \multirow{2}{*}{ Items } & \multirow{2}{*}{ Benefits of quitting } & \multicolumn{2}{|c|}{ Yes } & \multicolumn{2}{|c|}{ No } & \multicolumn{2}{|c|}{ Don't Know } \\
\hline & & No. & $\%$ & No. & $\%$ & No. & $\%$ \\
\hline $\mathrm{BQ}_{1}$ & Improves general health & 363 & 78.4 & 49 & 9.93 & 53 & 11.45 \\
\hline $\mathrm{BQ}_{2}$ & Health of people nearby are protected & 292 & 63.07 & 103 & 22.25 & 67 & 14.47 \\
\hline $\mathrm{BQ}_{3}$ & Reduces heart problem & 317 & 68.47 & 62 & 13.39 & 83 & 17.93 \\
\hline $\mathrm{BQ}_{4}$ & Reduces the respiratory problems & 304 & 65.66 & 61 & 13.17 & 97 & 20.95 \\
\hline $\mathrm{BQ}_{5}$ & Reduces the risk for cancer & 296 & 63.93 & 78 & 16.85 & 88 & 19 \\
\hline $\mathrm{BQ}_{6}$ & Heart attack will not occur & 268 & 57.88 & 74 & 15.98 & 120 & 25.93 \\
\hline $\mathrm{BQ}_{7}$ & Increases happiness in the family & 306 & 66.09 & 79 & 17.06 & 77 & 16.63 \\
\hline $\mathrm{BQ}_{8}$ & Paves way for saving money & 305 & 65.87 & 85 & 18.36 & 72 & 15.55 \\
\hline $\mathrm{BQ}_{9}$ & Removes vascular problems in leg & 164 & 35.42 & 73 & 15.77 & 225 & 48.6 \\
\hline $\mathrm{BQ}_{10}$ & Improves peace of mind & 257 & 55.51 & 110 & 23.76 & 95 & 20.52 \\
\hline
\end{tabular}


Table 4: Distribution of level of knowledge regarding benefits of quitting smoking ( $N=463)$.

\begin{tabular}{|c|c|c|c|}
\hline \multicolumn{2}{|c|}{ Knowledge on benefits of quitting } & \multicolumn{2}{|c|}{ Number } \\
\hline Score Range & Level of knowledge & 158 \\
\hline $0-3$ & Inadequate & 34.1 \\
\hline $6-\mathrm{Apr}$ & Moderate & 18.6 & 219 \\
\hline $10-\mathrm{Jul}$ & Adequate & 47.3 \\
\hline
\end{tabular}

Yang J et al. conducted study on health knowledge and perception of risks among Chinese smokers / non-smokers $(n=5956)$ [4]. The findings revealed that respondents were most likely to agree that smoking causes stained teeth and lung cancer followed by emphysema and lung cancer from second hand smoke. Only about $40 \%$ of the respondents agreed that smoking causes coronary heart disease, while one fifth agreed that smoking causes stroke and impotence. Only $7 \%$ of respondents agreed that smoking caused all kinds of the health effects [4].

Bhojani UM et al. findings on tobacco use and related factors among 300 pre-university students in a college in Bangalore showed the awareness about the harmful effects of tobacco use [5]. Of the 300 students, $78.3 \%$ reported that they were aware of the harmful effects of tobacco use. There was awareness regarding the ill-effects of tobacco use on health as cancer by $67 \%$ and respiratory illness by $38 \%$ of them. Only $4.4 \%$ of them had awareness regarding reproductive disorders [5].

Fu $\mathrm{M}$ et al. conducted a cross-sectional study to describe the nicotine dependence and readiness to quit smoking in the smoker population aged $>8$ years in Spain [6]. Telephone interviews were assessed with the Fagerstrom Test (FTND) and the readiness to quit using the Transtheoretical Model in a sample of cigarette smokers. Twenty-two percent of participants (95\% CI: 20.9-24.2\%) smoked an average of 14.4 cigarettes per day (standard deviation 9.15) and the mean FTND score was 2.8, with no differences by the stages of change. $64.3 \%$ (95\% CI: 60.3-68.2\%) of smokers were in the precontemplation stage, $25.4 \%$ (95\% CI: $21.8-28.9 \%$ ) in contemplation, and 10.4\% (95\% CI: 7.9-12.9\%) in preparation, with no differences by sex. The most nicotine dependent smokers (FTND>=6) had started to smoke at earlier ages, and smoked more cigarettes per day. Most of the Spanish smokers have low nicotine dependence and are in precontemplation stage. Smoking cessation programmes should be addressed to reduce dependence, help smokers to progress through the stages of change, and, consequently, reduce the prevalence of smokers in the population [6].

To help the smokers to quit, questions were framed to address the knowledge on benefits of quitting using 10 items scale in the present study. The level of knowledge regarding benefits of quitting was adequate among $219(47.3 \%)$ subjects, and $158(34.1 \%)$ had inadequate knowledge on benefits of quitting, and 86 (18.6\%) had moderate knowledge on benefits of quitting. Majority of the subjects had awareness that quitting smoking helps to improve the health of subjects and people around them, reduces heart problems (68.47\%), respiratory problems (65.66\%), and risk for cancer (63.93\%). There was lack of knowledge on reduction of vascular problems in legs $(64.37 \%)$ and the subjects were unaware of improvement in peace of mind.

Majority of the subjects had knowledge on the ill-effects of smoking such as cancer, lung problems, and heart attack, and so had clear understanding that quitting the smoking habit will help to reduce these problems. Basically, majority of the subjects had lack of knowledge on the ill-effects of smoking as vascular problems, impotence, and $4000^{+}$chemicals and so were unable to answer the benefit results from quitting smoking on these aspects.

In the present study most of the subjects were from rural area and the fact that the majority of them had education up to middle school level, contribute to lack of awareness about the ill-effects of smoking as $4000^{+}$chemicals, vascular problems, impotence etc. Few studies showed that the above mentioned findings on the ill-effects were not known even by college students and professionals. The findings highlight the need to increase awareness about health effects of smoking among the smokers to enable them to quit the habit. Apart from warning labels and mass education campaigns at larger level, while contacting smokers during hospitalization, providing health education with self help module may help the smokers to quit.

\section{Conclusion}

The findings of this study showed that smokers have low awareness level toward Hazards of smoking such as lung cancer to non-smokers from second-hand smoking, impotence in male smokers, and stroke. High level of education and high monthly income were associated with high knowledge score about the health effects of smoking. It's hoped that this study would be helpful for health policy-makers and health care professionals to understand the current knowledge and perception of smoking health effects. However, health care professionals should play an active role in communicating with their patients about the health effects of smoking to persuade smokers to quit smoking. In addition, increasing the taxes is also effective to reduce the consumption among smokers, especially those with lower socioeconomic status.

\section{References}

1. World Health Organization (2008) Global tobacco epidemic report. WHO Press, Geneva, Switzerland, pp. 328.

2. (2011) Global Health Observatory. World Health Organization.

3. Thankur JS, Lenka SR, Bhardwaj S, Kumar R. (2010). Why youth smoke? an exploratory community-based study from Chandigarh Union Territory of northern India. Indian J Cancer 47: 59-62.

4. Yang J, Hammond D, Driezen P, Fong GT, Jiang Y (2009) Health knowledge and perception of risks among Chinese smokers and non-smokers: findings from the Wave 1 ITC China Survey. Tobacco Control 19: 18-23.

5. Bhojani UM, Jahander, Devadessan (2009) A cross sectional study to understand tobacco use among 300 students from a preuniversity college in Bangalore. Indian J Med Res 67: 232-235.

6. Fu M, Martinez-Sanchez JM, Lopez MJ, Nebot M, Raich A, et al. (2011) Nicotine dependence and readiness to quit smoking in the Spanish population. Adicciones 23: 103-109. 